\title{
Fibroblast growth factor 23 and Klotho contribute to airway inflammation
}

\author{
Stefanie Krick ${ }^{1}$, Alexander Grabner ${ }^{2}$, Nathalie Baumlin ${ }^{3}$, Christopher Yanucil ${ }^{4}$, \\ Scott Helton ${ }^{1}$, Astrid Grosche ${ }^{3}$, Juliette Sailland ${ }^{3}$, Patrick Geraghty ${ }^{5}$, \\ Liliana Viera ${ }^{1}$, Derek W. Russell ${ }^{1}$, J. Michael Wells ${ }^{1,6}$, Xin Xu ${ }^{1}$, Amit Gaggar ${ }^{1}$, \\ Jarrod Barnes ${ }^{1}$, Gwendalyn D. King ${ }^{7}$, Michael Campos ${ }^{3}$, Christian Faul ${ }^{4,8}$ and \\ Matthias Salathe $e^{3,8}$
}

Affiliations: 'Division of Pulmonary, Allergy and Critical Care Medicine, Dept of Medicine, The University of Alabama at Birmingham, Birmingham, AL, USA. ${ }^{2}$ Division of Nephrology, Dept of Medicine, Duke University Medical Center, Duke University, Durham, NC, USA. ${ }^{3}$ Division of Pulmonary, Critical Care and Sleep Medicine, Dept of Medicine, University of Miami Leonard M. Miller School of Medicine, Miami, FL, USA. "Division of Nephrology, Dept of Medicine, The University of Alabama at Birmingham, Birmingham, AL, USA. ${ }^{5}$ Division of Pulmonary and Critical Care Medicine, Dept of Medicine, State University of New York Downstate Medical Center, Brooklyn, NY, USA. ${ }^{6}$ UAB Lung Health Center, Birmingham, AL, USA. ${ }^{7}$ Dept of Neurobiology, The University of Alabama at Birmingham, Birmingham, AL, USA. ${ }^{8}$ Both senior authors contributed equally to this work.

Correspondence: Stefanie Krick, Division of Pulmonary, Allergy and Critical Care Medicine, Dept of Medicine, The University of Alabama at Birmingham, 1900 University Boulevard, THT-422, Birmingham, AL 35294, USA. E-mail: skrick@uabmc.edu

\section{@ERSpublications}

Fibroblast growth factor 23 and cigarette smoke-induced FGFR4 signalling lead to the development of airway inflammation and emphysema http://ow.ly/j4tZ30jWyGF

Cite this article as: Krick S, Grabner A, Baumlin N, et al. Fibroblast growth factor 23 and Klotho contribute to airway inflammation. Eur Respir J 2018; 52: 1800236 [https://doi.org/10.1183/ 13993003.00236-2018].

ABSTRACT Circulating levels of fibroblast growth factor (FGF)23 are associated with systemic inflammation and increased mortality in chronic kidney disease. $\alpha$-Klotho, a co-receptor for FGF23, is downregulated in chronic obstructive pulmonary disease (COPD). However, whether FGF23 and Klothomediated FGF receptor (FGFR) activation delineates a pathophysiological mechanism in COPD remains unclear. We hypothesised that FGF23 can potentiate airway inflammation via Klotho-independent FGFR4 activation.

FGF23 and its effect were studied using plasma and transbronchial biopsies from COPD and control patients, and primary human bronchial epithelial cells isolated from COPD patients as well as a murine COPD model.

Plasma FGF23 levels were significantly elevated in COPD patients. Exposure of airway epithelial cells to cigarette smoke and FGF23 led to a significant increase in interleukin-1 $\beta$ release via Klotho-independent FGFR4-mediated activation of phospholipase $\mathrm{C} \gamma /$ nuclear factor of activated T-cells signalling. In addition, Klotho knockout mice developed COPD and showed airway inflammation and elevated FGFR4 expression in their lungs, whereas overexpression of Klotho led to an attenuation of airway inflammation.

Cigarette smoke induces airway inflammation by downregulation of Klotho and activation of FGFR4 in the airway epithelium in COPD. Inhibition of FGF23 or FGFR4 might serve as a novel anti-inflammatory strategy in COPD. 


\section{Introduction}

Chronic obstructive pulmonary disease (COPD) represents the third leading cause of global mortality; exposure to cigarette smoke is responsible for the majority of COPD cases and is the world's leading cause of avoidable premature mortality [1]. Various studies have shown inflammatory changes in the airway epithelium in COPD and due to cigarette smoke, including goblet hyperplasia and increased expression of pro-inflammatory cytokines [2-4].

Fibroblast growth factor (FGF)23, a $27-\mathrm{kDa}$ protein secreted by osteocytes, plays an essential role in maintaining serum phosphate homeostasis [5-7]. In chronic kidney disease, plasma concentrations of FGF23 are elevated and strongly associated with the risk of chronic kidney disease progression, cardiovascular events and mortality $[8,9]$. To date, direct effects of FGF23 on the lung have not been reported. However, it has been shown that Klotho expression is reduced in airways from COPD patients [10]. Klotho, a single-transmembrane protein that exists in a membrane-bound and soluble form, and acts as a co-receptor for FGF23, protects the alveolar epithelium against oxidative damage [11-13]. Furthermore, mice lacking Klotho develop an ageing phenotype with widened alveolar spaces, consistent with pulmonary emphysema [14].

FGF receptors (FGFRs) are a subfamily of receptor tyrosine kinases and consist of four isoforms (FGFR1-4) [15]. In its classic target organs, such as kidney and the parathyroid gland, FGF23 signals via FGFR1 and requires Klotho. By binding to FGFR1/Klotho, FGF23 activates the Ras/mitogen-activated protein kinase signalling pathway [16]. In the absence of Klotho, FGF23 can activate FGFR4 and induce subsequent phospholipase $\mathrm{C} \gamma$ (PLC $\gamma$ )/nuclear factor of activated T-cells (NFAT) signalling. This mechanism mediates pathophysiological actions of FGF23 that include the induction of hypertrophic growth in cardiac myocytes and the increased production of inflammatory cytokines in hepatocytes $[17,18]$.

We hypothesised that cigarette smoke contributes to increased production and activation of FGF23 signalling in COPD. Here, we examine whether cigarette smoke reduces Klotho and increases FGFR4 expression, resulting in pro-inflammatory PLC $\gamma /$ NFAT signalling in human primary bronchial epithelial cells (BECs). We postulate that FGF23 can potentiate inflammation in the lung via activation of FGFR4 and downregulation of Klotho.

\section{Methods}

Please refer to the supplementary material for further methodological details.

\section{Study approval}

The clinical study was conducted according to the principles of the Declaration of Helsinki and was approved by the Institutional Review Board of the University of Miami (Miami, FL, USA). Written informed consent was received from each participant prior to inclusion in the study. Human airways were obtained from organ donors whose lungs were rejected for transplant. Institutional Review Board-approved consent for research was obtained by the Life Alliance Organ Recovery Agency of the University of Miami or the LifeCenter Northwest (Bellevue, WA, USA) and conformed to the Declaration of Helsinki. All animal protocols were approved by the Institutional Animal Care and Use Committees at the University of Miami and The University of Alabama at Birmingham (Birmingham, AL, USA).

\section{Interleukin-1 $\beta,-6$ and -8 ELISA and mRNA assessment}

An ultrasensitive interleukin (IL)-1 $\beta$ and -6 ELISA from Invitrogen (Thermo Fisher Scientific, Scientific, Grand Island, NY, USA) was used. Human BECs were stimulated with FGF23 and/or cigarette smoke $\left(25 \mathrm{ng} \cdot \mathrm{mL}^{-1}\right.$ and/or 4 cigarettes for $24 \mathrm{~h}$ ) and $100 \mu \mathrm{L}$ of the basolateral medium (undiluted) was used for measurements. Gene expression was assessed by quantitative PCR using TaqMan probes (Life Technologies/Applied Biosystems, Carlsbad, CA, USA).

\section{Statistics}

Data were analysed with Prism version 5 (GraphPad, La Jolla, CA, USA) and are shown as mean \pm SEM using the t-test and ANOVA or the Kruskal-Wallis H-test with one-way ANOVA with appropriate post-tests for at least three independent experiments. Significance was accepted at $\mathrm{p}<0.05$.

\section{Results}

Plasma FGF23 levels are increased in individuals with COPD

We measured FGF23 in plasma samples from 28 individuals: 18 patients with COPD and 10 age-matched non-COPD patients. COPD was defined as forced expiratory volume in $1 \mathrm{~s}$ (FEV1)/forced vital capacity $<0.70$. Post-bronchodilator FEV1 \% pred was used to measure the severity of airflow obstruction, with FEV $1 \%$ pred $\geqslant 50 \%$ and $\leqslant 1$ COPD exacerbation within the last year indicating mild-to-moderate COPD 
(nine individuals) and $\mathrm{FEV}_{1} \%$ pred $<50 \%$ and $\geqslant 2$ COPD exacerbations within the last year indicating severe COPD (nine individuals). The clinical characteristics, including age, sex, smoking history and FEV 1 $\%$ pred, are shown in supplementary table S1. As expected, post-bronchodilator FEV1 \% pred was significantly lower in both COPD groups when compared with controls.

Individuals with mild-to-moderate COPD had higher FGF23 plasma levels when compared with patients without COPD (59 \pm 12 versus $42 \pm 10 \mathrm{pg} \cdot \mathrm{mL}^{-1}$; $\left.\mathrm{p}=0.004\right)$ (figure 1a). However, FGF23 was not significantly increased in severe COPD compared with controls in this study population $\left(51 \pm 18\right.$ versus $42 \pm 10 \mathrm{pg} \cdot \mathrm{mL}^{-1}$; $\mathrm{p}=0.26$ ). We therefore focused on mild-to-moderate COPD for further experimentation. We detected no difference between active smokers and nonsmokers (supplementary figure S1a).

Endobronchial biopsies from the mild-to-moderate subgroup with significantly elevated FGF23 levels showed BEC metaplasia (figure 1b, upper row). Tracheal sections from control and these COPD donors showed distinct FGF23 staining of the epithelial layer (figure 1b, lower row). As expected, the COPD group had significantly increased goblet cell numbers when compared with the control group (figure 1c).

We observed significant increases in plasma IL-6 levels (figure 1d, upper left panel) and bronchoalveolar lavage fluid (BALF) IL-8 levels (figure 1d, upper right panel) were also elevated in COPD patients. Determination of the Pearson correlation coefficient showed a significant positive correlation between plasma FGF23 levels and plasma IL-6 levels $\left(\mathrm{R}^{2}=0.2224\right.$ with $\left.\mathrm{p}=0.0415\right)$ (figure $1 \mathrm{~d}$, lower left panel), but no significant correlation between plasma FGF23 levels and BALF IL-8 levels $\left(\mathrm{R}^{2}=0.01542\right.$ with $\left.\mathrm{p}=0.7006\right)$ (figure 1d, lower right panel). Combined, these data suggest that COPD patients have increased plasma levels of FGF23, which coincide with elevated levels of inflammatory cytokines in the lung and circulation.

FGF23 and cigarette smoke induce IL-1 $\beta$ secretion in BECs from individuals with COPD

To determine direct effects of FGF23 in combination with cigarette smoke, we exposed primary BECs isolated from four different individuals with COPD (COPD-BECs) to cigarette smoke and FGF23, and assessed expression of IL- $6,-8$ and $-1 \beta$ by quantitative real-time PCR. Overall, we observed a considerable heterogeneity in cytokine expression. IL-6 mRNA increased with FGF23 and/or cigarette smoke with high variability (figure 2a); IL-8 was significantly increased with FGF23 and/or cigarette smoke, but the increase was less variable and more modest (figure $2 b$ ). IL- $1 \beta$ mRNA levels were not significantly increased by FGF23 alone, but the combination of both stimuli led to a greater than five-fold elevation (figure 2c). Exposure of COPD-BECs to either stimulus, however, caused a significant increase in basolateral secretion of IL-1 $\beta$, which was further elevated when both stimuli were combined (figure $2 \mathrm{~d}$ ). These findings indicate that, in combination with cigarette smoke, FGF23 can directly target COPD-BECs and induce the expression and release of IL-1 $\beta$.

\section{FGF23 activates FGFR4 and PLC $\gamma /$ calcineurin/NFAT signalling in COPD-BECs, resulting in} increased IL-1 $\beta$ secretion

As shown in other tissues, FGF23 can induce phosphorylation of both PLC $\gamma$ [19] and extracellular signal-regulated kinase (ERK) [20] depending on the absence or presence of Klotho. To determine whether FGF23 can activate FGFR4 and subsequent PLC $\gamma /$ calcineurin/NFAT signalling and induce an inflammatory response in the lung, as we have recently shown in the liver [18], we analysed FGFR4 binding to PLC $\gamma$ after treatment with FGF23.

In COPD-BECs, stimulation with FGF23 plus cigarette smoke caused phosphorylation of PLC $\gamma$, consistent with the premise that these effects might be mediated by Klotho-independent FGFR4 signalling. However, FGF23 alone did not result in phosphorylation of ERK, an effect clearly associated with cigarette smoke exposure (figure 3a, left panel).

In order to verify this finding, endogenous PLC $\gamma$ was immunoprecipitated and eluates analysed by immunoblotting with an anti-FGFR4 antibody. Compared with eluates from control cells, levels of copurified FGFR4 were elevated in FGF23-treated COPD-BECs, while total PLC $\gamma$ expression levels were unchanged. Pre-treatment with a FGFR4-specific blocking antibody (anti-FGFR4) [17] inhibited the co-immunoprecipitation of FGFR4 and PLC $\gamma$ (figure 3a, right panel). Combined exposure of COPD-BECs to FGF23 and cigarette smoke also led to the phosphorylation of PLC $\gamma$, which was blocked by anti-FGFR4 (figure 3b, left panel). Cigarette smoke, but not FGF23 stimulation by itself, caused a marked increase in ERK phosphorylation, which has been shown previously in BECs [21]. However, ERK phosphorylation was unchanged by pre-incubation with anti-FGFR4 (figure 3b, left panel). These findings indicate that FGF23 signalling in the bronchial epithelium occurs mainly via activation of PLC $\gamma$ rather than ERK and that this effect is mediated by FGFR4.

To determine if the FGFR4/PLC $\gamma /$ calcineurin/NFAT signalling cascade mediates FGF23 effects on IL-1 $\beta$ expression, we pre-incubated COPD-BECs with anti-FGFR4 followed by stimulation with FGF23 and 


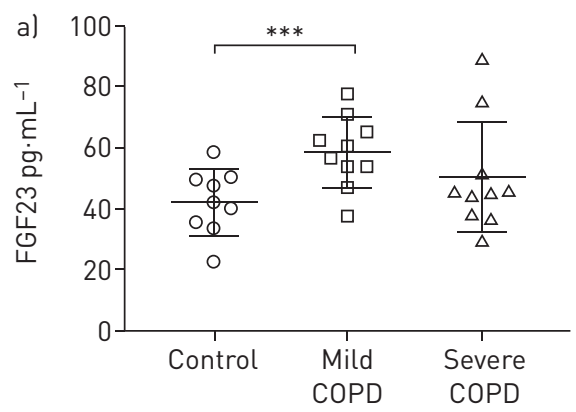

b)
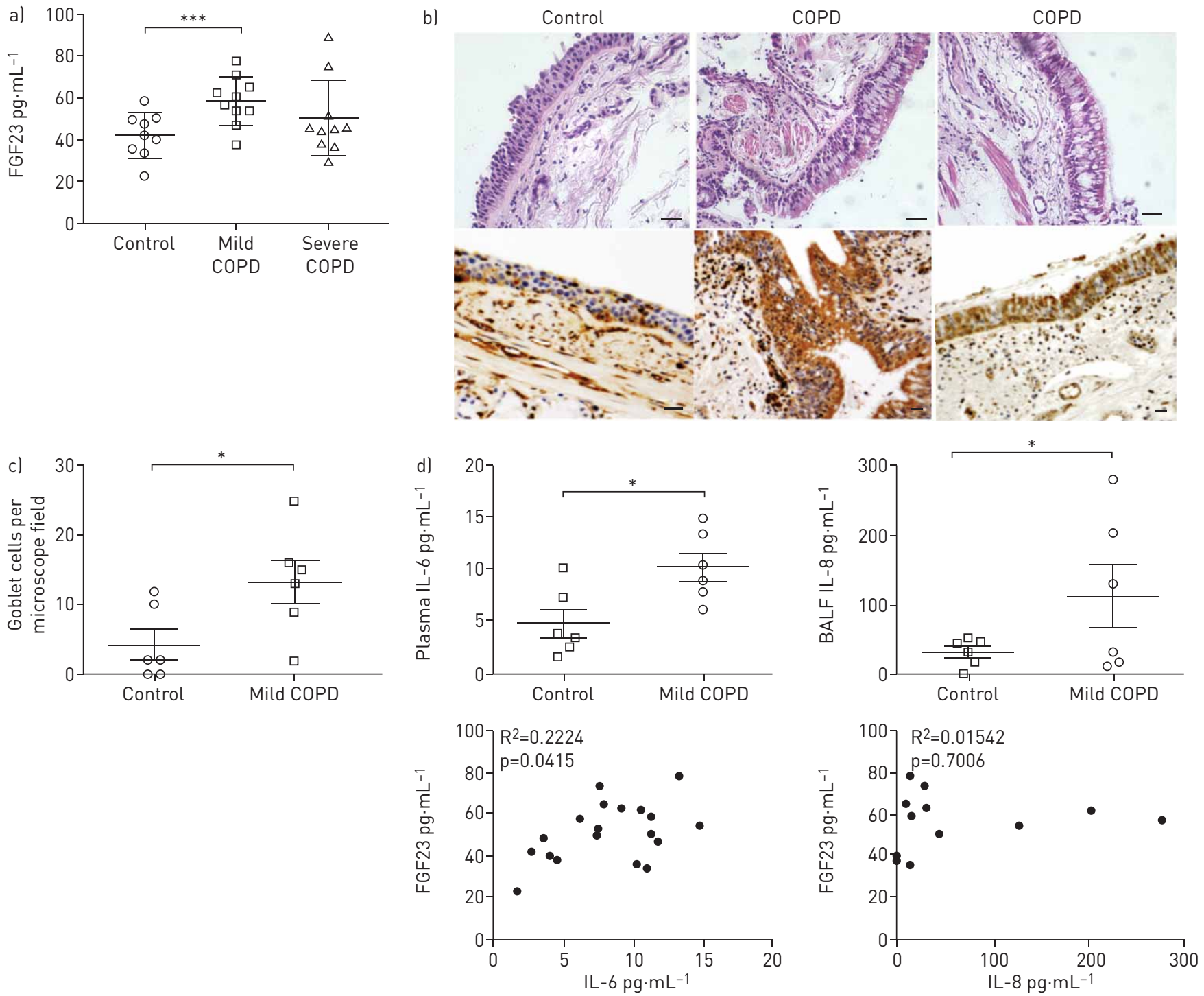

FIGURE 1 Fibroblast growth factor (FGF)23 levels are increased in patients with mild-to-moderate chronic obstructive pulmonary disease (COPD) who have goblet cell hyperplasia and increased levels of pro-inflammatory cytokines. IL: interleukin; BALF: bronchoalveolar lavage fluid. a) FGF23 plasma levels in non-COPD and COPD patients divided into mild-to-moderate ("mild") and severe COPD. b) Haematoxylin (upper row) and anti-FGF23 (lower row) staining of representative endobronchial biopsies from one control subject (left column) and two subjects (middle and right columns) with mild-to-moderate COPD. Scale bar: $40 \mu \mathrm{m}$. c) Quantification of goblet cells per field of view shows a significant increase in mild-to-moderate ("mild") COPD when compared with non-COPD subjects. $\mathrm{n}=6$ for each group. d) Plasma IL-6 levels (left panel) and BALF IL-8 levels (right panel), and dot-plots showing their correlation with FGF23 levels in control subjects and patients with mild-to-moderate ("mild") COPD. $n=6$ for each group. ${ }^{*}: p<0.05 ;{ }^{* * *}: p<0.005$.

cigarette smoke. Anti-FGFR4 significantly reduced elevations in mRNA as well as secreted levels of IL-1 $\beta$ (figure 3b, middle panel). To establish the link between FGF23 and NFAT activation, we employed the widely used calcineurin inhibitor cyclosporine A (CsA) [17, 18], and showed that CsA also inhibited the FGF23 and cigarette smoke-induced IL-1 $\beta$ responses (figure 3b, right panel). Furthermore, mRNA levels of cyclooxygenase (COX) 2, a NFAT target gene in the bronchial epithelium [22], were increased following co-stimulation with FGF23 and cigarette smoke. This effect was inhibited when cells were pre-treated with CsA (figure 3c, left panel). Additionally, we used the BEC line 16HBE and transfected these cells with a NFAT reporter gene-luciferase construct. An increase in NFAT activity was seen after stimulation with FGF23, which was significantly inhibited when cells were pre-incubated with anti-FGFR4 and CsA. Furthermore, cigarette smoke extract and cigarette smoke extract plus FGF23 also induced NFAT activation, which could be blocked by CsA (figure 3c, middle left panel).

We identified NFAT2c and NFAT3c as the most abundant isoforms in COPD-BECs, human BECs and $16 \mathrm{HBE}$ cells (figure $3 \mathrm{c}$, middle right and right panels). To further validate the specificity of 

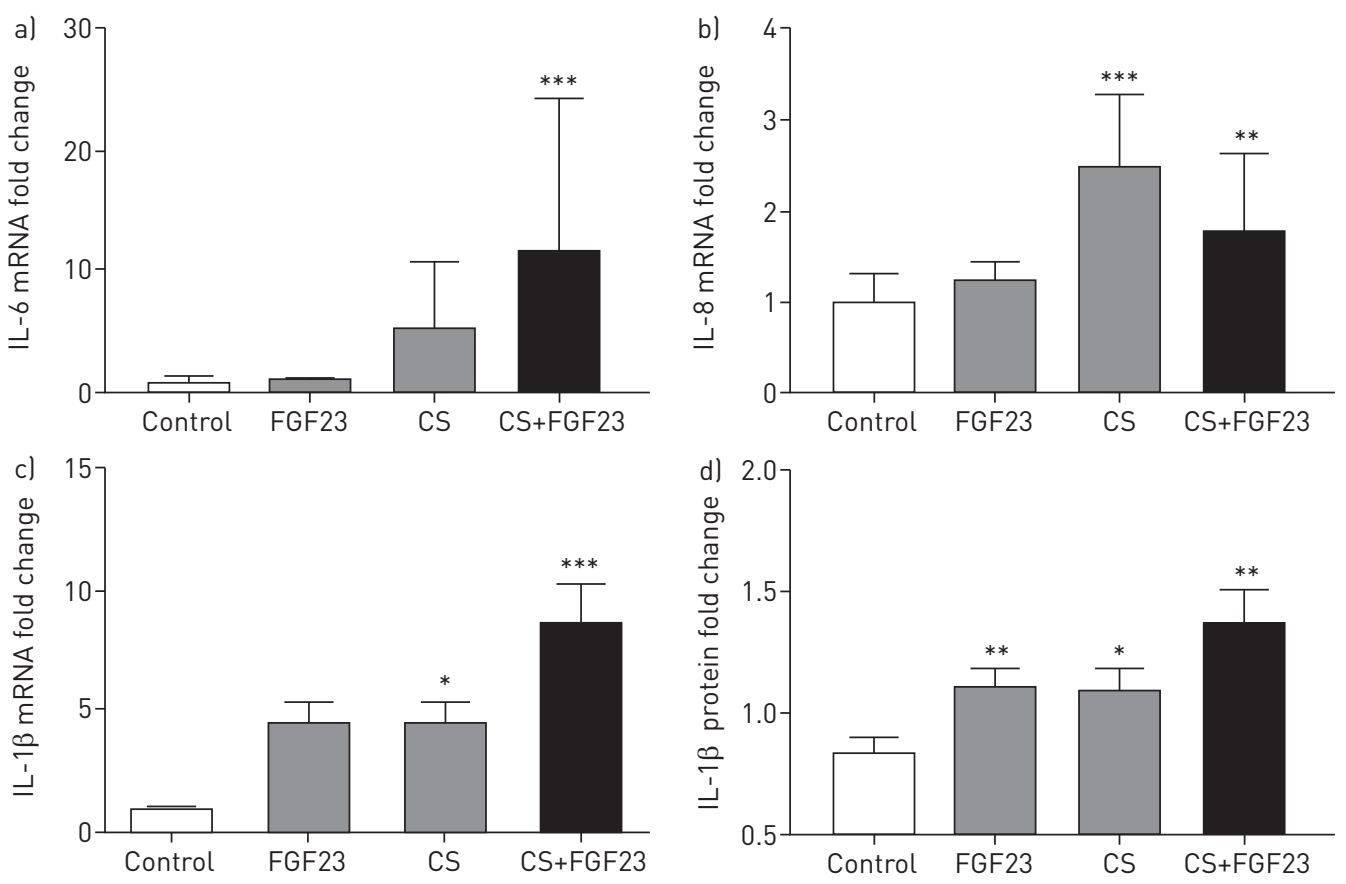

FIGURE 2 Effect of cigarette smoke (CS) and fibroblast growth factor (FGF)23 on expression of pro-inflammatory cytokines in chronic obstructive pulmonary disease (COPD) bronchial epithelial cells (BECs). a) Interleukin (IL)-6 and b) IL-8 mRNA levels in COPD-BECs $24 \mathrm{~h}$ after stimulation with CS (4 cigarettes) and/or FGF23 (25 ng. mL ${ }^{-1}$ ). c) IL-1 $\beta$ mRNA and d) IL-1 $\beta$ secreted protein levels in the basolateral media from COPD-BECs after stimulation with FGF23, CS or both. Data are represented as fold change in mRNA or protein expression with $n=3$ independent experiments from six different lungs. ${ }^{*}: p<0.05 ;{ }^{* *}: p<0.01$; ***: $p<0.005$, compared with control group.

FGF23-induced NFAT activation, both isoforms were knocked down by using small interfering (si)RNA in $16 \mathrm{HBE}$ cells (figure $3 \mathrm{~d}$, left panel). Since these $16 \mathrm{HBE}$ cells showed a similar IL-1 $\beta$ response as the primary cells (figure 3d, upper middle panel), we stimulated control, siNFAT2c and siNFAT3c transfected 16HBE cells with cigarette smoke extract and FGF23. siNFAT2c decreased both NFAT2c and NFAT3c, while siNFAT3c was isoform specific. Compared with "si control" transfected cells, the cigarette smoke extract and FGF23-induced IL-1 $\beta$ response was blunted in siNFAT2c and siNFAT3c transfected cells (figure 3d, lower middle panel). This indicates that both NFAT2c and NFAT3c are mediators of FGF23-induced signalling. Furthermore, cigarette smoke extract and/or FGF23 led to a significant increase in FGFR4 protein expression levels in $16 \mathrm{HBE}$ cells (figure 3d, right panel). Combined, our results show that FGF23 directly activates FGFR4/PLC $\gamma /$ calcineurin/NFAT signalling in BECs, thereby inducing IL- $1 \beta$.

\section{Exposure to cigarette smoke downregulates Klotho expression and upregulates FGFR4 in BECs from individuals with COPD}

Since Klotho expression is decreased in COPD airways [10], we analysed Klotho expression in a primary air-liquid interface BEC culture system. Exposure of COPD-BECs to cigarette smoke caused a significant decrease in Klotho protein and mRNA levels, whereas FGF23 alone had no effect (figure 4a). Compared with human BECs derived from nonsmoker and non-COPD lungs, Klotho mRNA and protein levels were significantly reduced in COPD-BECs (figure 4b). These data show that in COPD-BECs, Klotho expression is decreased and further reduced by cigarette smoke, indicating that both COPD and cigarette smoke might serve as factors that promote Klotho-independent FGF23 signalling in the lung.

We measured FGFR4 expression in the lung, as FGFR4 has been previously shown to mediate the Klotho-independent FGF23 signalling in the heart and liver [17, 18]. Paraffin-embedded endobronchial biopsies were used for haematoxylin staining and FGFR4 immunohistochemistry. FGFR4 staining was localised to the bronchial epithelium and appeared to be increased in endobronchial tissue biopsies from COPD patients (figure 4c, left panel). To better quantify FGFR4 expression, FGFR4 mRNA levels were determined and showed a significant increase in COPD-BECs compared with control human BECs (figure $4 \mathrm{c}$, right panel).

Next, COPD-BECs were exposed to an increasing number of cigarette puffs. A dose-dependent upregulation of FGFR4 protein and mRNA levels was detected after $24 \mathrm{~h}$ (figure $4 \mathrm{~d}$ ). Taken together, 
a)

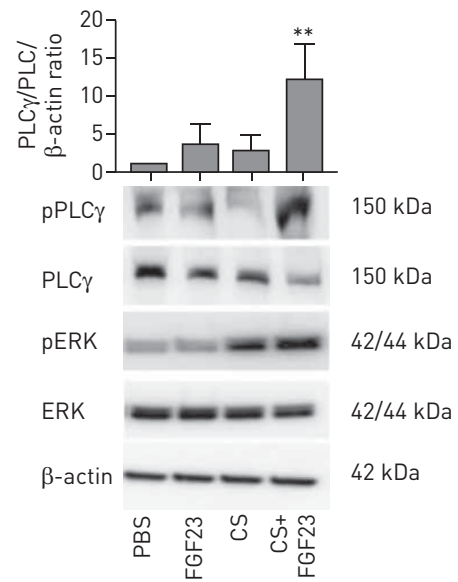

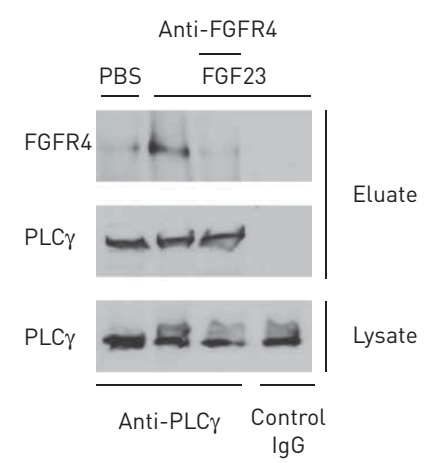

b)
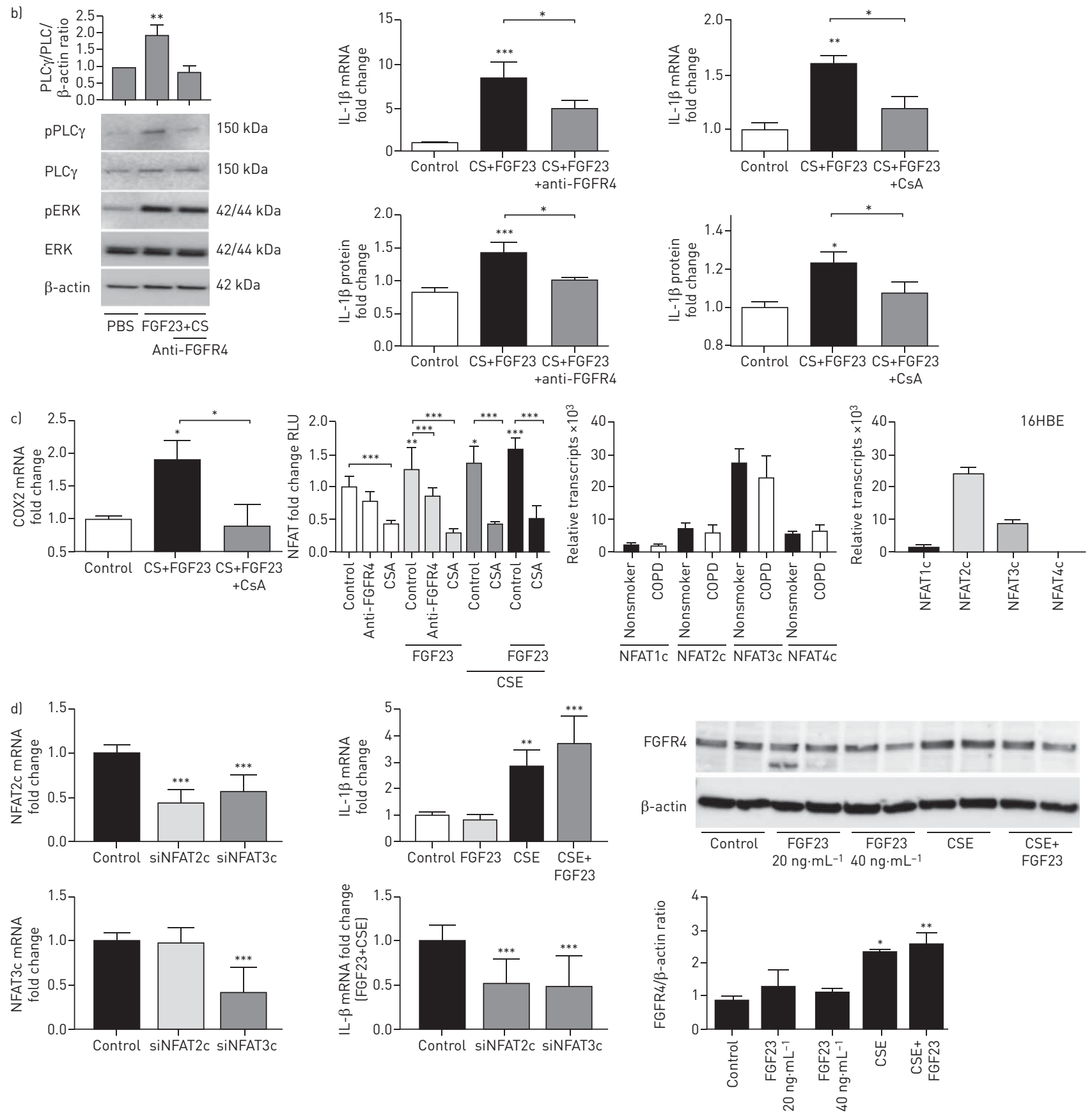

FIGURE 3 Caption on following page. 
FIGURE 3 Fibroblast growth factor (FGF)23 induces activation of FGF receptor 4 (FGFR4)/phospholipase $\mathrm{C} \gamma$ (PLC $\gamma$ )/nuclear factor of activated T-cells (NFAT) signalling in chronic obstructive pulmonary disease (COPD) bronchial epithelial cells (BECs). p: phosphorylated; ERK: extracellular signal-regulated kinase; CS(E): cigarette smoke (extract); IL: interleukin; CsA: cyclosporine A; COX: cyclooxygenase; RLU: relative luciferase units; si: small interfering. a) Left panel: representative immunoblot and densitometric analysis (pPLC $\gamma /$ total PLC $\gamma / \beta$-actin ratio) show FGF23 plus CS-induced phosphorylation of PLC $\gamma$ (FGF23 and CS dependent) and of ERK (purely CS dependent). n=3. Right panel: immunoblot of co-immunoprecipitation with FGFR4 using an anti-PLC $\gamma$ antibody shows activation of PLC $\gamma$ after FGF23 stimulation in COPD-BECs, which could be inhibited by pre-incubation with anti-FGFR4. b) CS plus FGF23 induces PLC $\gamma$ and ERK phosphorylation as seen in (a), but anti-FGFR4 only inhibits PLC $\gamma$ phosphorylation. Both anti-FGFR4 and CsA, a calcineurin inhibitor, inhibit CS plus FGF23-mediated induction of IL-1 $\beta$ mRNA and secreted protein levels. c) CsA also attenuates induction of COX2 mRNA. FGF23, CSE alone and both activate NFAT, as assessed by a luciferase-based reporter gene assay in 16HBE cells, which can be blocked by CsA. Anti-FGFR4 inhibits FGF23-induced NFAT activation. The right two bar graphs indicate mRNA levels of NFAT isoforms in both human BECs from nonsmokers and COPD patients as well as 16HBE cells. d) siRNAs targeting NFAT isoforms NFAT2c and NFAT3c were transfected into $16 \mathrm{HBE}$ cells. siNFAT2c reduces the expression of NFAT2c and NFAT3c, whereas siNFAT3c is specific for NFAT3c (left panel). When 16HBE cells were stimulated with FGF23 (20 ng. $\left.\mathrm{mL}^{-1}\right), \mathrm{CSE}(5 \%)$ or both for $24 \mathrm{~h}$, IL-1 $\beta$ increased (middle panel). Finally, the effects of siNFAT2c (knockdown of both the $2 \mathrm{c}$ and $3 \mathrm{c}$ isoforms) and siNFAT3c transfection were assessed after stimulation with CSE plus FGF23 (right panel). CSE and/or FGF23 also led to significant upregulation of FGFR4 protein expression in $16 \mathrm{HBE}$ cells. All $n=3$ independent experiments (from three different lungs in the case of COPD-BECs). ${ }^{*}: p<0.05 ;{ }^{* *}: p<0.01 ;{ }^{* * *}: p<0.005$.

FGFR4 is expressed in the bronchial epithelium and upregulated in COPD patients and upon exposure to cigarette smoke.

\section{Klotho deficiency leads to increased FGFR4 activation and inflammation}

As genetic mouse models lacking Klotho develop elevated serum FGF23 levels, we wanted to determine if Klotho-independent FGF23 signalling and associated inflammation occur in the lungs of these animals. As shown by others previously [12], we observed widened airway spaces on haematoxylin and eosin staining by immunohistochemical characterisation of Klotho hypomorphic mice $\left(\mathrm{kl}^{-/-}\right)$(figure $5 \mathrm{a}$ ).

Mean linear intercept analysis was used to quantify airspace enlargement, which was significantly higher in Klotho-deficient mouse lungs compared with wild-type $\left(\mathrm{kl}^{+/+}\right)$littermates (figure $5 \mathrm{~b}$, left upper panel). Furthermore, FGF23 mRNA levels were upregulated in $\mathrm{kl}^{-/-}$lungs when compared with $\mathrm{kl}^{+/+}$(figure 5b, left lower panel). Immunohistochemical analysis of lung sections using anti-FGF23 showed an intense signal in the bronchial epithelium (figure 5b, right panel). When compared with their wild-type littermates, BALF from $\mathrm{kl}^{-1-}$ mice contained more cells, specifically neutrophils [23] and macrophages/ monocytes (figure $5 \mathrm{c}$ ). There was no difference in the number of lymphocytes between both groups, but IL-6 mRNA levels were also increased (figure 5c). To determine the activity of FGFR4/PLC $\gamma$, we immunoprecipitated PLC $\gamma$ from mouse lung tissue extracts followed by immunoblotting of eluates for FGFR4. We found an increase in co-purified FGFR4 in lungs from $k^{-1-}$ mice when compared with wild-type littermates (figure 5d, left panel). Furthermore, FGFR4 mRNA levels were increased in cultured, fully differentiated murine tracheal epithelial cells (MTECs) from $\mathrm{kl}^{-/-}$mice (figure $5 \mathrm{~d}$, middle panel). They also showed a significantly increased basolateral secretion of IL-6 (figure 5d, right panel). These findings indicate that in the absence of Klotho and in the presence of elevated FGF23, FGFR4/PLC $\gamma$ signalling and production of inflammatory cytokines are elevated in the mouse lung.

\section{Soluble Klotho protects COPD-BECs from pro-inflammatory effects of FGF23 and cigarette smoke}

The extracellular domain of Klotho can exist in a soluble form [24]. It has been shown that soluble Klotho has protective, anti-inflammatory effects on different cell types $[25,26]$. To test if soluble Klotho interferes with the effects of FGF23 in the lung, we co-incubated COPD-BECs with FGF23, cigarette smoke and the ectodomain of recombinant human $\alpha$-Klotho at $0.1 \mu \mathrm{g} \cdot \mathrm{mL}^{-1}$ for $30 \mathrm{~min}$. As shown earlier, FGF23 and cigarette smoke caused an increase in PLC $\gamma$ phosphorylation without affecting total PLC $\gamma$ levels. In the presence of FGF23 and cigarette smoke, treatment of COPD-BECs with soluble Klotho also led to a decrease in IL-1 $\beta$ secretion (figure $6 b$ ).

\section{Overexpression or knockdown of Klotho in mice alters FGF23/FGFR4 signalling}

Since mice that were exposed to chronic cigarette smoke [27] did not show an upregulation of FGF23 serum levels (supplementary figure S1b), we used an acute mouse model for cigarette smoke exposure. Mice were exposed to 1 week of cigarette smoke to induce acute airway inflammation. Western blot analyses of total lung tissue showed an increase in FGF23 levels in some mice (six out of 11 cigarette smoke-exposed mice and three out of 11 control mice) (figure 7a). However, there was no significant increase in serum FGF23 levels between the control and cigarette smoke-exposed groups (figure 7a), and there was no significant difference in mRNA levels of Klotho or FGFR4 in lung tissue (figure 7a). When comparing wild-type mice and Klotho overexpressing mice [28], the latter group showed increased Klotho mRNA levels in the lung, which was independent of cigarette smoke exposure (figure $7 \mathrm{~b}$, left panel). We detected no difference in serum FGF23 levels and in BALF total cell count (figure 7b, middle panels). Smoke-induced increase of IL- 6 in the lung was decreased in the Klotho overexpressing mice when 

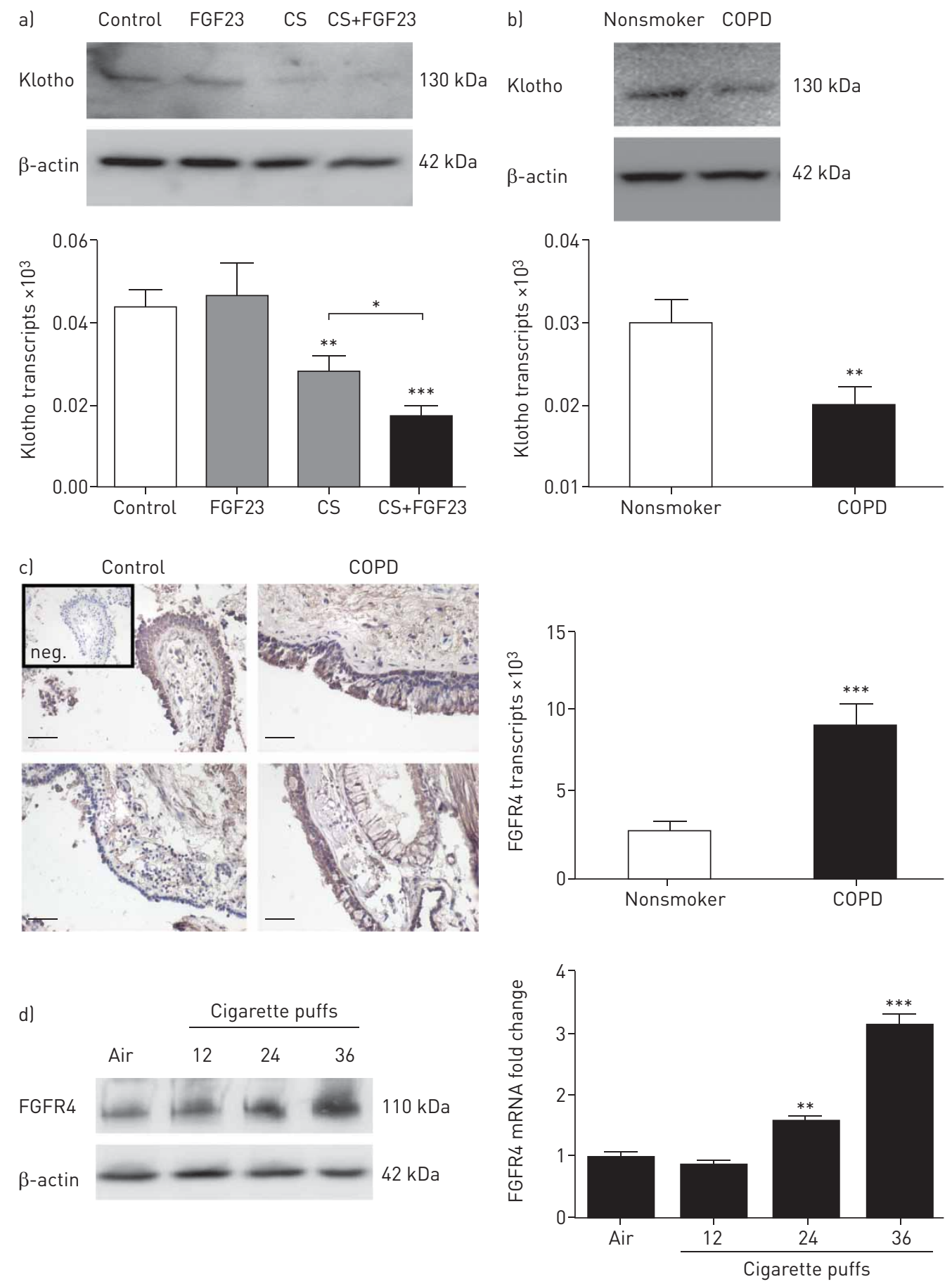

FIGURE 4 Cigarette smoke (CS) exposure regulates expression of Klotho and fibroblast growth factor (FGF) receptor 4 (FGFR4) in chronic obstructive pulmonary disease (COPD) airway epithelia. BEC: bronchial epithelial cell. a) Representative immunoblot showing downregulation of Klotho protein and bar graph indicating mRNA levels from COPD-BECs after treatment with CS, FGF23 or both stimuli for $24 \mathrm{~h}$. b) Representative immunoblot and Klotho mRNA levels of human BECs from nonsmokers compared with COPD-BECs. $n=3$ independent experiments from six different lungs. c) Left: immunohistochemistry for FGFR4 and counterstaining with haematoxylin of endobronchial biopsies from two representative control patients and two COPD patients, compared with a negative control (inset: "neg."). Scale bar: $50 \mu \mathrm{m}$. Right: bar graph showing increased FGFR4 mRNA expression in COPD-BECs compared with control lungs. d) Immunoblot analysis of FGFR4 protein levels (representative blot from one COPD patient, tested in three different COPD lungs) and quantitative real-time PCR of FGFR4 mRNA levels in COPD-BECs $24 \mathrm{~h}$ after exposure to 2, 4 and 6 cigarettes (1 cigarette $=6$ puffs). All $n=3$ independent experiments from three different lungs. ${ }^{*}: p<0.05$; ${ }^{* *}: p<0.01 ;{ }^{* * *}: p<0.001$. 
a)

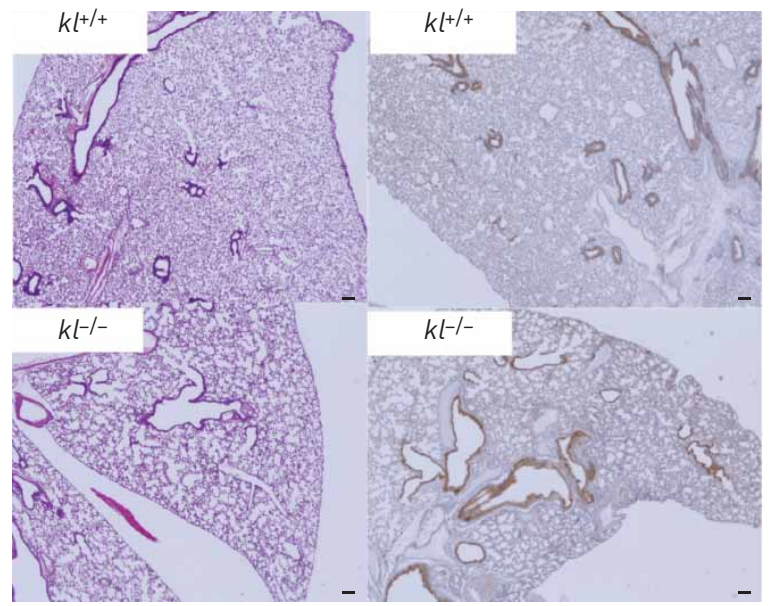

c)
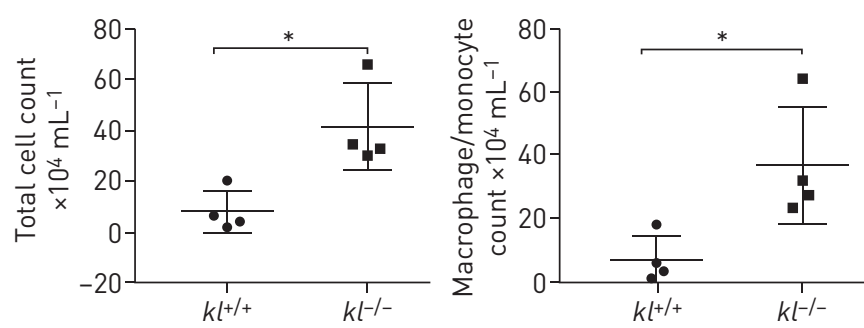
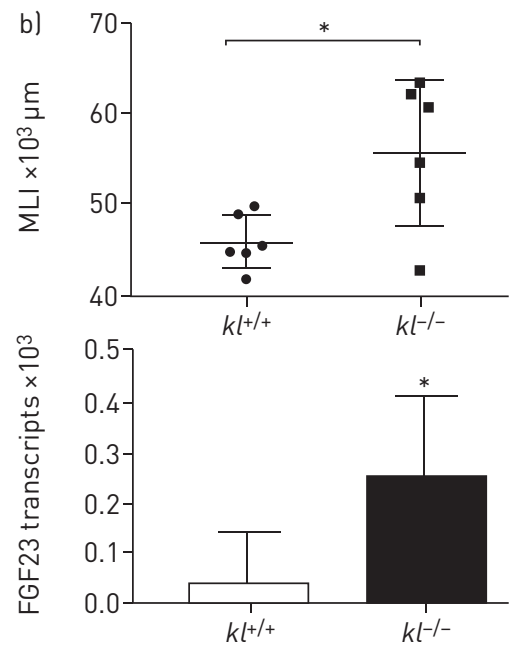
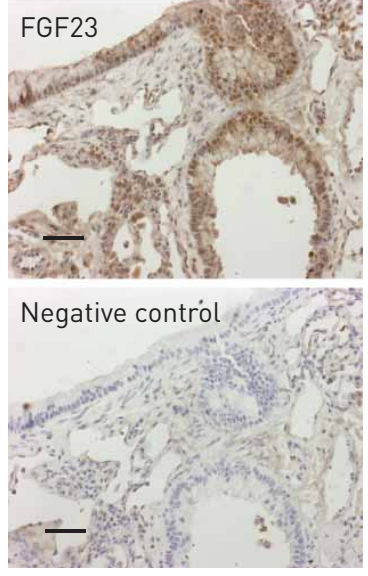
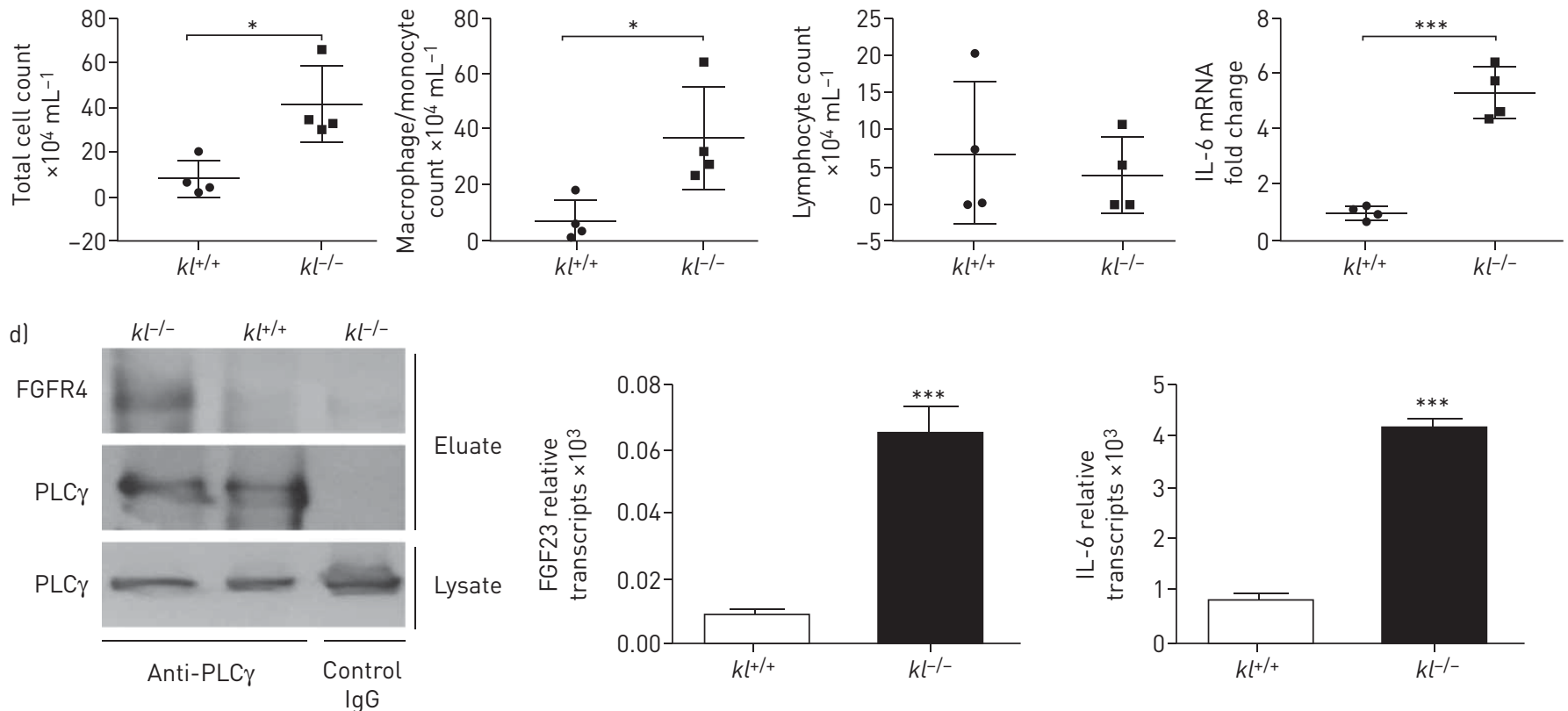

FIGURE 5 Loss of Klotho expression causes increased airway inflammation in mice. MLI: mean linear intercept; FGF(R): fibroblast growth factor (receptor); BALF: bronchoalveolar lavage fluid; MTEC: murine tracheal epithelial cell; IL: interleukin; PLC $\gamma$ : phospholipase C $\gamma$. al Haematoxylin and anti-FGF23 staining of lung tissue from wild-type $\left(\mathrm{kl}^{+/+}\right)$and hypomorphic Klotho $\left(\mathrm{kl}^{-/-}\right)$mice. Scale bar: $50 \mu \mathrm{m}$. b) Left panels: MLI analysis demonstrates widened airspaces in the $\mathrm{kl}^{-1-}$ lung samples and FGF23 mRNA levels are upregulated in whole lung tissue from $\mathrm{kl}^{-1-}$ when compared with $\mathrm{kl}^{+/+}$mice. $\mathrm{n}=6$ mice for each group. Right panels: immunohistochemical staining using anti-FGF23 in paraffin-embedded mouse lung tissue sections indicates a signal in the bronchial epithelium. Scale bar: $50 \mu \mathrm{m}$. c) Assessment of total cells in BALF from $\mathrm{kl}^{+/+}$versus $\mathrm{kl}^{-1-}$ mice. $\mathrm{n}=4$ mice for each group. Also shown are BALF macrophage/monocyte count, BALF lymphocyte count and IL-6 fold change in BALF from $\mathrm{kl}^{+/+}$versus $\mathrm{kl}^{-/-}$mice. d) Co-immunoprecipitation of endogenous FGFR4 by using anti-PLC $\gamma$ or anti-Green Fluorescent Protein (control) in lung tissue lysates from either $\mathrm{kl}^{-/-}$or $\mathrm{kl}^{+/+}$mice indicates increased FGFR4 transcripts in $\mathrm{kl}^{-l_{-}}$lungs. Quantitative real-time PCR for FGFR4 mRNA in $\mathrm{kl}^{-l-}$ MTECs showed a significant upregulation when compared with wild-type MTECs and IL-6 protein levels in basolateral media from $\mathrm{kl}^{+/+}$and $\mathrm{kl}^{-l-}$ MTECs. For mouse experiments, three different independent experiments were done; for each experiment, cells were pooled from $\mathrm{n}=3-4$ animals; morphometry and BALF analysis were done from $n=4$ mice in each group. ${ }^{*}: p<0.05 ;{ }^{* *}: p<0.001$.

compared with wild-type littermates (figure $7 \mathrm{~b}$, right panel). To test the effect of Klotho deficiency, and since $\mathrm{kl}^{-{ }^{--}}$mice were too sick to be exposed to cigarette smoke, MTECs were isolated from both $\mathrm{kl}^{+/+}$and $\mathrm{kl}^{-/-}$mice. At baseline, $\mathrm{kl}^{-/-}$MTECs had higher mRNA levels of IL-6 and FGFR4 when compared with wild-type cells (figure 7c).

To further characterise the link between Klotho deficiency, FGF23 increase and elevated FGFR4 expression as direct or indirect, MTECs from FGFR4 constitutively active transgenic mice were analysed and did not show any difference in IL-6 levels compared with control MTECs. Interestingly, there was also a trend toward an increase in Klotho mRNA levels in these FGFR4 transgenic MTECs (supplementary figure 
a)

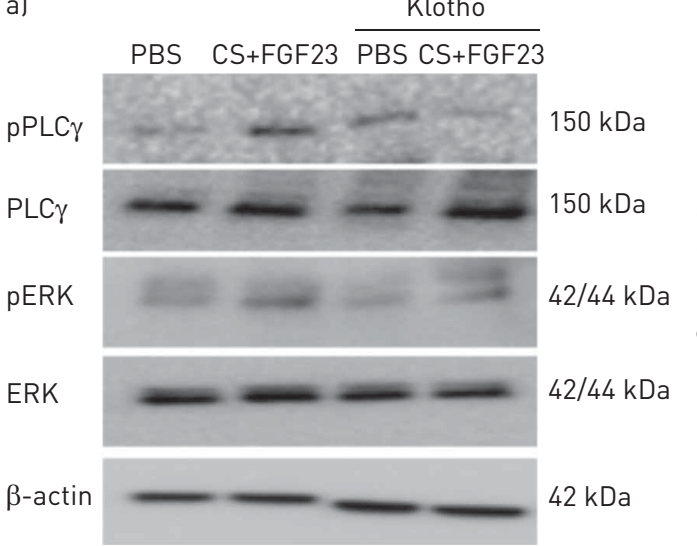

b)

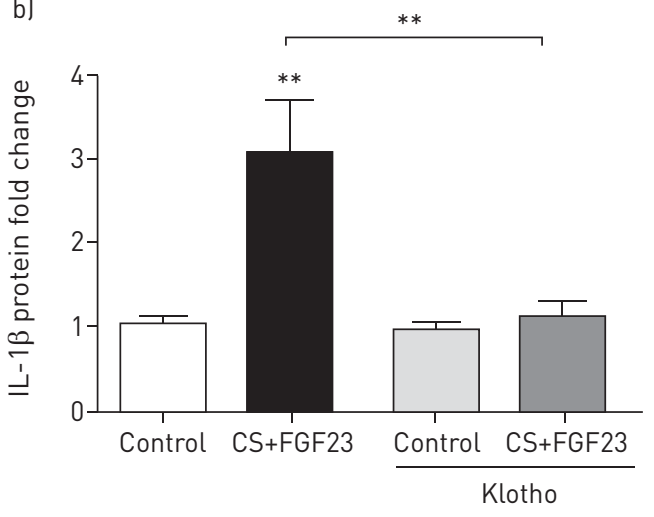

FIGURE 6 Supplementation of human recombinant Klotho inhibits cigarette smoke (CS) and fibroblast growth factor (FGF)23-induced interleukin (IL)-1 $\beta$ secretion. $p$ : phosphorylated; PLC $\gamma$ : phospholipase C $\gamma$; ERK: extracellular signal-regulated kinase. a) Representative immunoblots show FGF23 ( $25 \mathrm{ng} \cdot \mathrm{mL}^{-1}$ for $30 \mathrm{~min}$ ) induced phosphorylation of PLC $\gamma$. Inhibition of PLC $\gamma$ phosphorylation occurs by pre-incubation with Klotho $\left(1 \mu \mathrm{g} \cdot \mathrm{mL}^{-1}\right.$, lane $3 ; 0.1 \mu \mathrm{g} \cdot \mathrm{mL}^{-1}$, lane 4). b) Soluble Klotho inhibits CS and FGF23-mediated increases of IL-1 $\beta$ protein levels, assessed by ELISA from basolateral media. All $n=3$ independent experiments from three different lungs. ${ }^{* *}: p<0.01$.

S2a and b). MTECs from $f g f r 4^{-/-}$mice though showed protection from FGF23 and cigarette smoke-induced IL-6 increase accompanied by a downregulation of Klotho expression (supplementary figure S2c and d).

In summary, our findings indicate that in COPD there is cigarette smoke-induced upregulation of FGFR4 expression and a decrease in soluble Klotho, enabling elevated FGF23 to signal via FGFR4/PLC $\gamma /$ NFAT to

a)
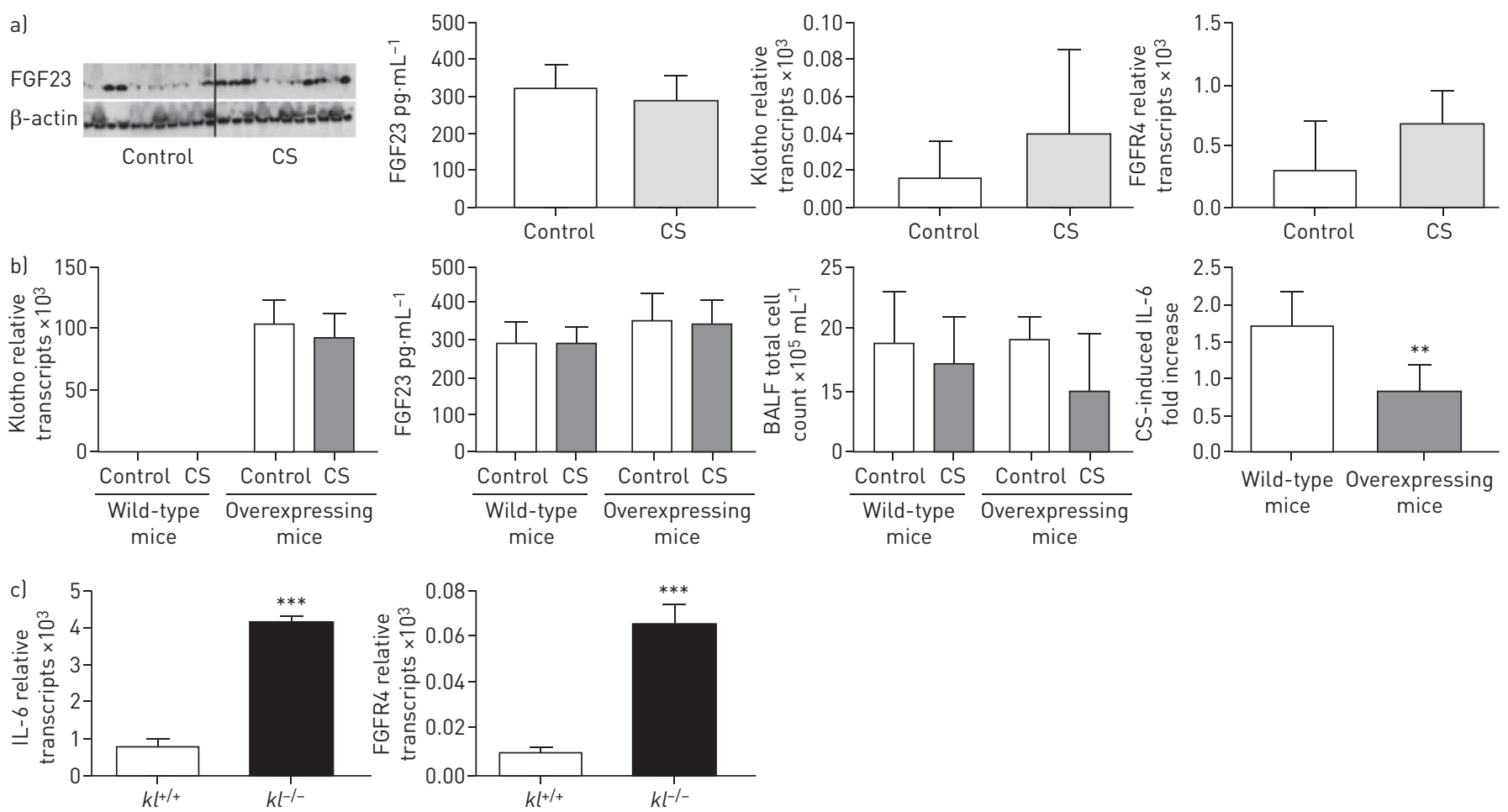

FIGURE 7 Effect of Klotho overexpression and Klotho deficiency on fibroblast growth factor (FGF)23 signalling in mice. CS: cigarette smoke; FGFR4: FGF receptor 4; BALF: bronchoalveolar lavage fluid; IL: interleukin. a) Representative immunoblots show FGF23 protein levels in total lung lysates from control mice and mice acutely exposed to CS. Bar graphs show serum FGF23 levels, total lung Klotho and FGFR4 mRNA levels in both mouse groups. b) Klotho mRNA levels, serum FGF23 levels, BALF total cell count and CS-induced fold increase in total mouse lung IL-6 expression after exposure to acute CS and compared with nonexposed control mice (both wild-type and Klotho overexpressing mice). c) IL-6 and FGFR4 transcript levels of murine tracheal epithelial cells isolated from wild-type $\left(k l^{+/+}\right)$and hypomorphic Klotho $\left(k l^{-l-}\right)$ mice. ${ }^{* *}: p<0.01 ;{ }^{* * *}: p<0.005$. 


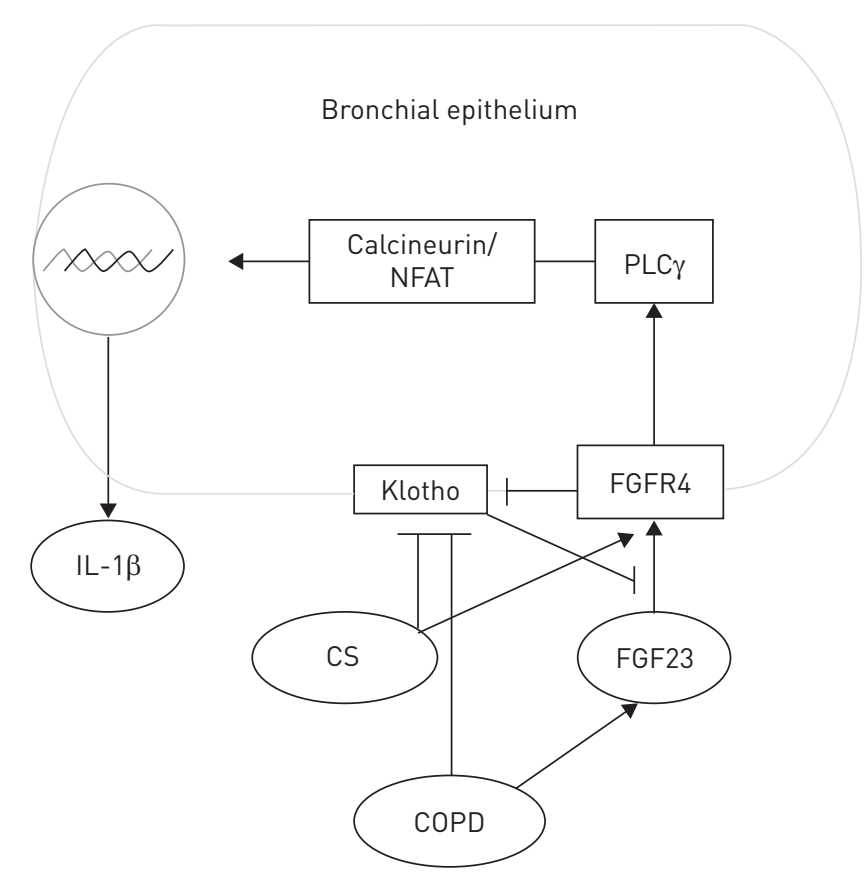

FIGURE 8 Possible signalling mechanism for cigarette smoke (CS) and fibroblast growth factor (FGF) 23-mediated regulation of FGF receptor 4 (FGFR4)/phospholipase $\mathrm{C} \gamma(\mathrm{PLC} \gamma)$ /calcineurin/nuclear factor of activated T-cells (NFAT) pathway. Diagram illustrating the direct effects of FGF23 and CS on the chronic obstructive pulmonary disease (COPD) airway epithelium: CS induces upregulation of FGFR4 and decreases Klotho expression in the bronchial epithelium. Elevated FGF23 plasma levels activate FGFR4/PLC $\gamma /$ calcineurin/NFAT signalling to release interleukin (IL)-1 $\beta$, thereby inducing inflammation.

induce airway inflammation (figure 8). Furthermore, co-administration with soluble Klotho can block FGF23-mediated signalling in COPD-BECs, thereby inhibiting the pro-inflammatory actions of FGF23.

\section{Discussion}

Here, we show for the first time FGF23-induced signalling in the bronchial epithelium and provide new insights into the opposing roles of FGF23 and its co-receptor Klotho in cigarette smoke-induced chronic bronchitis. We demonstrate that 1) plasma FGF23 levels are elevated in patients with mild-to-moderate COPD and an associated inflammatory phenotype with goblet cell hyperplasia, 2) cigarette smoke exposure in combination with FGF23 induces IL-1 $\beta$ secretion from primary human BEC cultures from COPD patients, 3) cigarette smoke induces an increase of FGFR4 and a decrease of Klotho expression, thereby causing activation of PLC $\gamma /$ NFAT signalling and inflammation, and 4) the presence of soluble Klotho attenuates cigarette smoke-induced IL-1 $\beta$ secretion.

A positive correlation between plasma FGF23 levels and smoking has been described previously using a multivariable regression analysis of 604 patients with chronic kidney disease [29]. However, in that study the smokers were not further characterised regarding COPD. In a different report, increased FGF23 levels in COPD patients were linked to hypophosphataemia, but a mechanism for elevation and the source of circulating FGF23 was not identified [30]. Here, we show that FGF23 levels appear not to correlate with disease severity per se, since there is no systemic FGF23 elevation in patients with severe COPD. One explanation could be that FGF23/Klotho perturbations may decrease in advanced disease due to "burn-out" as the residual mass of viable lung epithelium decreases. If this is the case, FGF23/Klotho dysregulation may effectively represent a marker not only of disease but also for "at-risk" lung tissue mass. Therefore, we focused on the group of these patients with mild-to-moderate COPD by assessing inflammation in their plasma and transbronchial biopsy specimens. Klotho, a co-receptor for FGF23, is downregulated in airway epithelial cells from COPD patients [10]. In addition, previous reports showed that gene silencing of Klotho in a BEC line induces IL-8 secretion that is further enhanced by cigarette smoke extract [10].

Our data also show that in a COPD mouse model (due to deficiency of Klotho) there is increased bronchial inflammation, which is at least partially generated by the BECs and an influx of macrophages and neutrophils. We further characterise the underlying novel signalling pathway for cigarette 
smoke-induced inflammatory airway disease that has the potential for future therapeutic intervention, i.e. FGFR4 activation and consecutive PLC $\gamma /$ calcineurin/NFAT signalling, which occurs in a state of Klotho deficiency and upregulation of FGF23. It is challenging to experimentally characterise the link between Klotho deficiency and our data indicate that regulation of both is tightly linked. It has been shown previously that Klotho knockout mice have elevated FGF23 levels [19]. Currently, it is not clear how soluble Klotho blocks the FGF23 effects in BECs, since ERK phosphorylation is not altered. However, we showed that soluble Klotho is not a circulating co-receptor for FGF23 in the lung that mediates ERK signalling. Therefore, further studies are needed.

Interestingly, we also observed ERK activation in cigarette smoke-induced IL-1 $\beta$ expression, which appears to be FGF23/FGFR4 independent, as there was no change after pre-incubation with a FGFR4 inhibitor (figure 3b). Since cigarette smoke downregulates Klotho expression, FGF23 signalling occurs via FGFR4, as we have previously demonstrated in the liver and in the myocardium $[17,18]$. This ultimately leads to an increase in IL-1 $\beta$ secretion, a key cytokine in virus-induced lung disease [31]. Administration of an IL-1 $\beta$ antibody reduces lung inflammation in a mouse model of influenza [32]. Furthermore, IL-1 $\beta$ is elevated in total lung tissue and sputum from COPD patients [33], and is associated with frequent COPD exacerbations as well as an IL-1 $\beta$-associated sputum proteomic signature $[34,35]$.

Identifying new COPD-related signalling pathways is important to develop novel future therapeutic strategies. FGFRs are ubiquitously expressed, whereas FGFR4 is mainly found in lung, heart, kidney and liver. Signalling via FGFR4 is associated with inflammatory changes, and we demonstrate several links of FGFR4 signalling to airspace remodelling and inflammation. In addition, a FGFR4 blocking antibody and small-molecule inhibitors have been developed for the treatment of hepatocellular carcinomas [36, 37]. Therefore, therapeutically targeting FGFR4 or soluble Klotho levels in the inflammatory subtypes of COPD may represent novel therapeutic options.

Acknowledgements: The authors thank J.S. Dennis (University of Miami, Miami, FL, USA), D. Duncan and S. Hutcheson (The University of Alabama at Birmingham, Birmingham, AL, USA) for technical assistance. They also thank G. Holt and E. Donna (University of Miami, Miami, FL, USA) for assisting in obtaining the bronchoscopic specimen used in this study, and R. Abraham and J. Bange (U3 Pharma, Martinsried, Germany) for providing the anti-FGFR4 blocking antibody.

Conflict of interest: None declared.

Support statement: This work was supported by the Flight Attendant Medical Research Institute (YFAC152003 to S. Krick, YCSA113380 to P. Geraghty and CIA13033 to M. Salathe), the Cystic Fibrosis Foundation (CFF KRICK1610 to S. Krick and SALATH14G0 to M. Salathe), the American Heart Association (A. Grabner and C. Faul), the American Diabetes Association (C. Faul), the James and Esther King Florida Biomedical Research Program (5JK02 to M. Salathe) and the National Institutes of Health (R01HL128714 to C. Faul). Funding information for this article has been deposited with the Crossref Funder Registry.

\section{References}

1 Samet JM. Tobacco smoking: the leading cause of preventable disease worldwide. Thorac Surg Clin 2013; 23: $103-112$.

2 de Boer WI, Sont JK, van Schadewijk A, et al. Monocyte chemoattractant protein 1, interleukin 8, and chronic airways inflammation in COPD. J Pathol 2000; 190: 619-626.

3 Polosukhin VV. Ultrastructural of the bronchial epithelium in chronic inflammation. Ultrastruct Pathol 2001; 25: $119-128$

4 Mortaz E, Henricks PA, Kraneveld AD, et al. Cigarette smoke induces the release of CXCL-8 from human bronchial epithelial cells via TLRs and induction of the inflammasome. Biochim Biophys Acta 2011; 1812: $1104-1110$

5 Schiavi SC, Kumar R. The phosphatonin pathway: new insights in phosphate homeostasis. Kidney Int 2004; 65: $1-14$.

6 Mirams M, Robinson BG, Mason RS, et al. Bone as a source of FGF23: regulation by phosphate? Bone 2004; 35: 1192-1199.

7 Benet-Pages A, Lorenz-Depiereux B, Zischka H, et al. FGF23 is processed by proprotein convertases but not by PHEX. Bone 2004; 35: 455-462.

8 Gutierrez OM, Mannstadt M, Isakova $\mathrm{T}$, et al. Fibroblast growth factor 23 and mortality among patients undergoing hemodialysis. N Engl J Med 2008; 359: 584-592.

9 Shimada T, Urakawa I, Isakova $\mathrm{T}$, et al. Circulating fibroblast growth factor 23 in patients with end-stage renal disease treated by peritoneal dialysis is intact and biologically active. J Clin Endocrinol Metab 2010; 95: 578-585.

10 Gao W, Yuan C, Zhang J, et al. Klotho expression is reduced in COPD airway epithelial cells: effects on inflammation and oxidant injury. Clin Sci 2015; 129: 1011-1023.

11 Ravikumar P, Ye J, Zhang J, et al. $\alpha$-Klotho protects against oxidative damage in pulmonary epithelia. Am J Physiol Lung Cell Mol Physiol 2014; 307: L566-L575.

12 Kuro-o M, Matsumura $\mathrm{Y}$, Aizawa $\mathrm{H}$, et al. Mutation of the mouse klotho gene leads to a syndrome resembling ageing. Nature 1997; 390: 45-51.

13 Chen G, Liu Y, Goetz R, et al. $\alpha$-Klotho is a non-enzymatic molecular scaffold for FGF23 hormone signalling. Nature 2018; 553: 461-466. 
14 Suga T, Kurabayashi M, Sando Y, et al. Disruption of the klotho gene causes pulmonary emphysema in mice. Defect in maintenance of pulmonary integrity during postnatal life. Am J Respir Cell Mol Biol 2000; 22: 26-33.

15 Faul C. Cardiac actions of fibroblast growth factor 23. Bone 2017; 100: 69-79.

16 Urakawa I, Yamazaki Y, Shimada T, et al. Klotho converts canonical FGF receptor into a specific receptor for FGF23. Nature 2006; 444: 770-774.

17 Grabner A, Amaral AP, Schramm K, et al. activation of cardiac fibroblast growth factor receptor 4 causes left ventricular hypertrophy. Cell Metab 2015; 22: 1020-1032.

18 Singh S, Grabner A, Yanucil C, et al. Fibroblast growth factor 23 directly targets hepatocytes to promote inflammation in chronic kidney disease. Kidney Int 2016; 90: 985-996.

19 Faul C, Amaral AP, Oskouei B, et al. FGF23 induces left ventricular hypertrophy. J Clin Invest 2011; 121: 4393-4408.

20 Olauson H, Lindberg K, Amin R, et al. Parathyroid-specific deletion of Klotho unravels a novel calcineurin-dependent FGF23 signaling pathway that regulates PTH secretion. PLoS Genet 2013; 9: e1003975.

$21 \mathrm{Xu} \mathrm{X}$, Balsiger R, Tyrrell J, et al. Cigarette smoke exposure reveals a novel role for the MEK/ERK1/2 MAPK pathway in regulation of CFTR. Biochim Biophys Acta 2015; 1850: 1224-1232.

22 Ding J, Li J, Xue C, et al. Cyclooxygenase-2 induction by arsenite is through a nuclear factor of activated T-cell-dependent pathway and plays an antiapoptotic role in Beas-2B cells. J Biol Chem 2006; 281: $24405-24413$.

23 Krick S, Baumlin N, Aller SP, et al. Klotho inhibits interleukin-8 secretion from cystic fibrosis airway epithelia. Sci Rep 2017; 7: 14388.

$24 \mathrm{Hu}$ MC, Shi M, Zhang J, et al. Klotho deficiency causes vascular calcification in chronic kidney disease. J Am Soc Nephrol 2011; 22: 124-136.

25 Blake DJ, Reese CM, Garcia M, et al. Soluble extracellular Klotho decreases sensitivity to cigarette smoke induced cell death in human lung epithelial cells. Toxicol In Vitro 2015; 29: 1647-1652.

$26 \mathrm{Li} \mathrm{L}$, Wang $\mathrm{Y}$, Gao W, et al. Klotho reduction in alveolar macrophages contributes to cigarette smoke extract-induced inflammation in chronic obstructive pulmonary disease. J Biol Chem 2015; 290: 27890-27900.

27 Foronjy RF, Salathe MA, Dabo AJ, et al. TLR9 expression is required for the development of cigarette smoke-induced emphysema in mice. Am J Physiol Lung Cell Mol Physiol 2016; 311: L154-L166.

28 Li Q, Vo HT, Wang J, et al. Klotho regulates CA1 hippocampal synaptic plasticity. Neuroscience 2017; 347: 123-133.

29 Vervloet MG, van Zuilen AD, Heijboer AC, et al. Fibroblast growth factor 23 is associated with proteinuria and smoking in chronic kidney disease: an analysis of the MASTERPLAN cohort. BMC Nephrol 2012; 13: 20.

30 Elsammak MY, Attia A, Suleman M. Fibroblast growth factor-23 and hypophosphatemia in chronic obstructive pulmonary disease patients. J Med Biochem 2012; 31: 12-18.

31 Kim KS, Jung H, Shin IK, et al. Induction of interleukin-1 beta (IL-1 $\beta$ ) is a critical component of lung inflammation during influenza A (H1N1) virus infection. J Med Virol 2015; 87: 1104-1112.

32 Indalao IL, Sawabuchi T, Takahashi E, et al. IL-1 $\beta$ is a key cytokine that induces trypsin upregulation in the influenza virus-cytokine-trypsin cycle. Arch Virol 2017; 162: 201-211.

33 Pauwels NS, Bracke KR, Dupont LL, et al. Role of IL- $1 \alpha$ and the Nlrp3/caspase-1/IL-1 $\beta$ axis in cigarette smoke-induced pulmonary inflammation and COPD. Eur Respir J 2011; 38: 1019-1028.

$34 \mathrm{Fu}$ JJ, McDonald VM, Baines KJ, et al. Airway IL-1 $\beta$ and systemic inflammation as predictors of future exacerbation risk in asthma and COPD. Chest 2015; 148: 618-629.

35 Damera G, Pham TH, Zhang J, et al. A sputum proteomic signature that associates with increased IL-1beta levels and bacterial exacerbations of COPD. Lung 2016; 194: 363-369.

36 Hagel M, Miduturu C, Sheets M, et al. First selective small molecule inhibitor of FGFR4 for the treatment of hepatocellular carcinomas with an activated FGFR4 signaling pathway. Cancer Disc 2015; 5: 424-437.

37 Ho HK, Pok S, Streit S, et al. Fibroblast growth factor receptor 4 regulates proliferation, anti-apoptosis and alpha-fetoprotein secretion during hepatocellular carcinoma progression and represents a potential target for therapeutic intervention. J Hepatol 2009; 50: 118-127. 